This is a postprint version of the following published document:

Pozuelo, J. \& Baselga, J. (2002): Glass transition temperature of low molecular weight poly(3-aminopropyl methyl siloxane). A molecular dynamics study. Polymer, 43 (22), pp.: 6049-6055.

DOI: $\underline{10.1016 / \mathrm{S} 0032-3861(02) 00442-1}$

(C) Elsevier, 2002

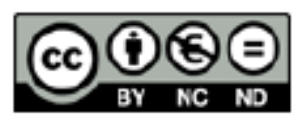

This work is licensed under a Creative Commons Attribution-NonCommercialNoDerivatives 4.0 International License. 


\title{
Glass transition temperature of low molecular weight poly(3-aminopropyl methyl siloxane). A molecular dynamics study
}

\author{
Javier Pozuelo*, Juan Baselga \\ Instituto Tecnológico de Química y Materiales, Universidad Carlos III de Madrid, 28911 Leganés, Madrid, Spain
}

\begin{abstract}
The average specific volume of the model poly(3-aminopropyl methyl siloxane) as a function of temperature near the glass transition was computed from molecular dynamics simulations. The glass transition temperature was defined as the slop intersection around $210 \mathrm{~K}$, a value similar to that of the experimental result. Globular polymer shaped chains were observed where the chain is closed upon itself. Three amino groups of amino propylene chains were located in the center and the rest of the amino groups were situated outside the main chain. The glass transition temperature of this low molecular weight polymer strongly depends on the binding energies between chains. The intersection of binding energy slopes defines a temperature of

$213 \mathrm{~K}$ near the glass transition temperature. The most important contributions to the glass transition changes were the electrostatic binding contributions. The Van der Waals contributions in the volume changes were less important. The chain mobility was evaluated by the transition between angles for the states trans, $g^{+}$and $g$. The glass transition temperature observed experimentally, $208 \pm 2 \mathrm{~K}$, is due to cooperative movements of two different torsion angles, (O Si) and ( $\mathrm{Si} \mathrm{C}$ ) of the main chain and the lateral chain, respectively, and its rotational mobility. Self-diffusion constant variation for all polymer atoms with the temperature is a probe that the polymer chain cooperative movement had started at temperatures around the glass transition temperature.
\end{abstract}

Keywords: Molecular dynamics; Glass transition temperature; Siloxane

\section{Introduction}

Linear functionalized polysiloxanes constitute an interesting and not very well studied family of reactive polymers. They may serve as model compounds to understand the properties of coupling regions in fiber reinforced polymer composites. For example, glass fibers that have been used as reinforcement for polymer matrix composite materials are usually surface treated with ethoxy silanes that have vinyl, epoxy or amino groups [1]. Such monomers react with the glass surface and also among themselves to form a graded crosslinked polymer layer [2,3] with a high surface density of reactive groups. In subsequent processes, such groups may react with the polymer matrix, thus coupling the composite constituents chemical and mechanically. The structure and properties of these interfacial regions has been extensively studied [2 7] although it remains an open issue.

Of special interest is the thermal behavior of these polymers, since mechanical composite properties may depend on the coupling layer interfacial flexibility. By means of a fluorescent labeling technique, the authors were able to study the low temperature relaxation on poly(3-aminopropyl methyl siloxane) (PAMS) chemisorbed over glass fibers [8]. Measuring the shift of the 00 vibronic transition of the pyrenil mobility attached to the polymer and the exciplex ratio as a function of temperature, at 186 and $204 \mathrm{~K}$, two transitions were obtained. The origin of these transitions should depend on the polymer nature and on the interactions between the label and the lateral groups of the main chain.

To study isolated poly(3-aminopropyl methyl siloxane) is extremely difficult because it tends to give highly crosslinked networks without the restrictions imposed by the surface of the glass. Therefore, the bulk and chemisorbed polymer present different properties. Considering the reported graded 3D structure of the coupling region, it seems that a more realistic model for the chemisorbed polymer should be oligomeric linear polymers like poly(3aminopropyl methyl siloxane).

In this work we will study the thermal behavior of PAMS by molecular dynamics simulations. Using the NPT 
ensemble molecular dynamics method it is possible to evaluate the equilibrium volume of a model system at constant pressure and temperature. Hence, it enables us to predict glass transition temperatures and thermal expansion coefficients directly from simulations performed at different temperatures. Earlier studies showed that even from short time molecular dynamics simulations (100 ps), the glass transition temperature could be obtained for poly(ethylene) and poly(propylene) [9 12]. More recent studies used a much longer simulation time for a number of amorphous polymers, including poly(ethylene), atactic poly(propylene), atactic poly(styrene), cis-poly(1,3-butadiene), and poly(isobutylene) [13 17] and polycarbonates [18]. Furthermore, molecular dynamics simulations at the atomistic level have the important advantage that they can provide detailed information regarding chain dynamics and structure associated to the system temperature. Hence, the simulations are particularly useful for revealing patterns, time scales, and energies associated with the different molecular motion modes in the vicinity of the glass transition temperature.

In this work a correlation between the molecular motion and thermal behavior of poly(3-aminopropyl methyl siloxane) will be established. Constant pressure and temperature molecular dynamics (NPT) simulations lasting $2 \mathrm{~ns}$ will be performed in the vicinity of the glass transition temperature. After locating the glass transition temperature for the model polymer from volumetric changes, both the local chain motion and the conformational transitions below and above the glass transition temperature will be analyzed in great detail and the interaction energies between chains will bequantified. Finally, the revision of some commonly accepted concepts related to cooperativity as an origin of the glass transition temperature [19] will be proposed.

\section{Molecular dynamics simulations (MD) methodology}

The molecule studied by MD was built according to the synthesized structure described by Cabanelas et al. [38]. It consisted of a linear poly(3-aminopropyl methyl siloxane) polymer with 20 repeat units. The molecule was end capped with two hydroxyl groups as determined by ${ }^{29} \mathrm{Si} \mathrm{NMR}$. The atactic configuration was selected with a ratio between meso and racemic dyads of 50\%. The initial structure was carrier out in two steps:

1. Initial conformation was made according to the Theodorou and Suter technique [20]. This consisted of the generation of an initial chain configuration using a rotational isomeric state/Monte Carlo scheme, followed by an energy minimization step to relax the structure to mechanical equilibrium. These initial structures had fixed covalent bond lengths and valence angles. Temperature enters the procedure only in setting the system density and statistical weights used in the RIS generation. As initial density a value of $1 \mathrm{~g} \mathrm{~cm}^{1}$ was taken.
2. To optimize both structure and energy, a MD simulation was made with fixed NVT and variable bond lengths, bond angles and torsion angles. $T$ was increased from 0 to $2000 \mathrm{~K}$ in steps of $50 \mathrm{~K}$ every 2 ps of interval duration. Afterwards, $T$ was decreased to $0 \mathrm{~K}$ following exactly the same temperature and time profile. With this procedure, one equilibrated initial conformation was obtained.

The PCFF 91 force field was used in all simulations. With this force field, the energy is computed as a sum of 13 energy contributions:

$E \quad E_{\mathrm{str}}+E_{\mathrm{bond}}+E_{\mathrm{tor}}+E_{\mathrm{Wil}}+E_{\mathrm{VdW}}+E_{\mathrm{ele}}+E_{\mathrm{cross}}$

where the terms on the right hand side represent the contribution from bond stretching, bond angle bending, torsion, Van der Waals, electrostatic interactions, Wilson (or umbrella out-of-plane) and seven crossterms. This force field with a 69 Van der Waals contribution, uses partial charges optimized for this polymer type as well as a dielectric constant of the unity with a good behavior.

The molecular dynamics trajectories were computed using Cerius2 4.2 and the PCFF 91 force field [21 28]. Calculations were performed in a canonical ensemble, with NPT fixed but allowing bond lengths, bond angles and torsion angles to vary during the simulation. Constant pressure and temperature were maintained using the extended Hamiltonian formalism according to the works of Andersen [29] and Parrinello and Rahman [30] for pressure and Nosé and Hoover [31 33] for temperature. A value of 0.1 ps was taken for the time constant [34], $\tau$, of the thermostat mass parameter $Q \quad 3 N k T \tau^{2}$ of the Nosé model. External pressure was set at $1 \mathrm{~atm}(\approx 0.0001 \mathrm{GPa})$. As cutoff for non-bonded interactions between 9 and $14 \AA$ a spline switching function [35] was used. The duration of each trajectory was 2 ns computed with a time step of $1 \mathrm{fs}$. The images were saved every 0.2 ps yielding 10,000 images from each simulation for subsequent analysis. Six temperatures around the experimental glass transition temperature were used on the Molecular dynamics simulations 173, 183, $193,203,213,218$ and $223 \mathrm{~K}$. The average of any property $\langle X\rangle$ was obtained as:

$$
\langle X\rangle \quad \frac{1}{N} \sum_{i} X_{i}
$$

Where $N$ is the number of images and $X_{i}$ is the value of the property for image $i$.

For each image, the non-bonded interaction between polysiloxane chains, denoted $E_{\text {binding, }}$, was evaluated as the difference between the total potential energy of the 3Dmatrix $\left(E_{\text {matrix }}\right)$ and that corresponding to isolated chains $\left(E_{\text {isolated chain }}\right)$

$E_{\text {binding }} \quad E_{\text {matrix }} \quad E_{\text {isolated-chain }}$

The self-diffusion constant was obtained using the 


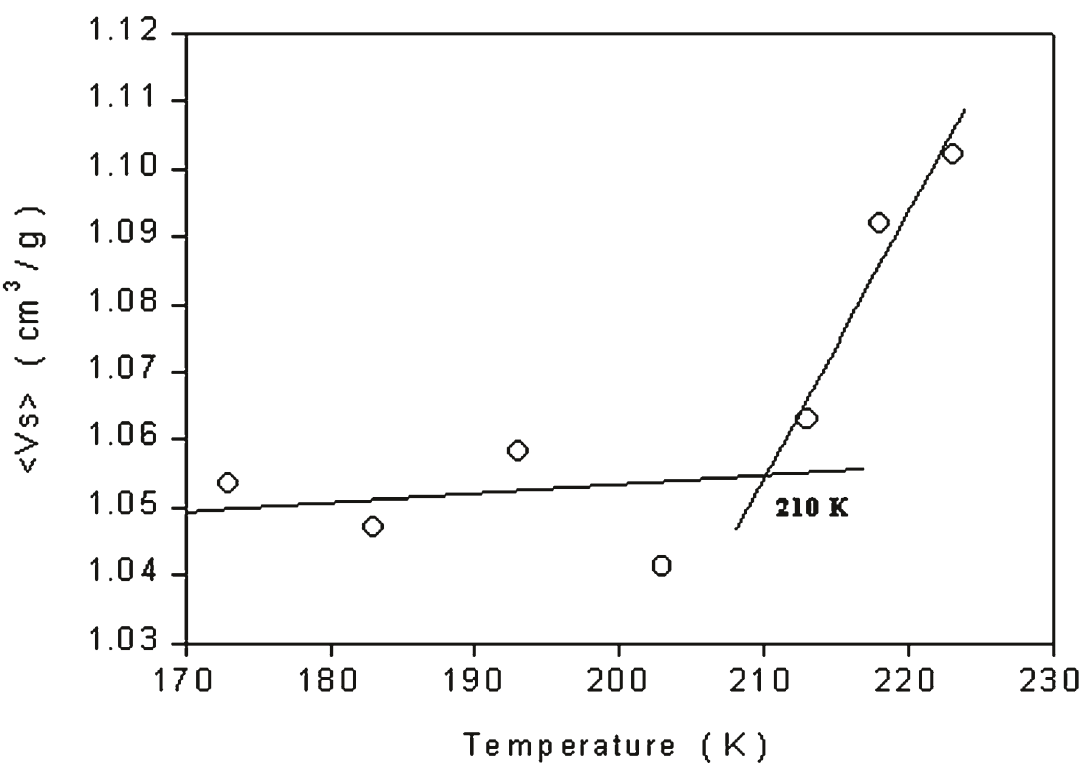

Fig. 1. Specific volume of poly(3 aminopropyl methyl siloxane) as a function of temperature.

Einstein relation:

$D \quad \frac{1}{6 N t}\left\langle\left|r(t) \quad r^{2}\right|\right\rangle$

Where $N$ is the number atoms, $r$ the position of the particle and $t$ the time. The expression within the braces is the meansquare displacement (MSD). The self-diffusion constant $(D)$ is obtained by calculating the slope of MSD vs. time.

\section{Results and discussion}

\subsection{Volume relaxation and glass transition temperature}

Fig. 1 shows the average specific volume of poly(3aminopropyl methyl siloxane) as a function of temperature, obtained from MD simulations. The figure shows two different linear regions around $210 \mathrm{~K}$. The volumetric thermal expansion coefficients $(\alpha)$ in these two regions were estimated from the slopes resulting in values of $(1 \pm 3) \times 10^{4}$ and $(4 \pm 1) \times 10^{3} \mathrm{~K}$, respectively. Below the intersection at $210 \mathrm{~K}$, the $\alpha$ value was similar to that of common glass systems, around $10{ }^{4} \mathrm{~K} \quad{ }^{1}$. Above $210 \mathrm{~K}, \alpha$ increases by one

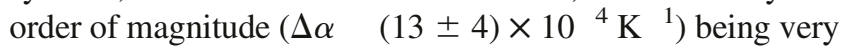
similar to rubbery systems [36]. The $\alpha$ value above $210 \mathrm{~K}$ as

Table 1

End to end distance, radius of gyration and characteristic ratio of the poly(3 aminopropyl methyl siloxane) at seven temperatures

\begin{tabular}{llll}
\hline$T(\mathrm{~K})$ & $\left\langle r^{2}\right\rangle^{1 / 2}(\AA)$ & $\left\langle s^{2}\right\rangle^{1 / 2}(\AA)$ & $C_{N}$ \\
\hline 173 & 12.3 & 8.5 & 2.6 \\
183 & 12.2 & 8.4 & 2.6 \\
193 & 12.9 & 8.2 & 2.8 \\
203 & 12.5 & 8.2 & 2.7 \\
213 & 13.8 & 8.5 & 3.2 \\
218 & 14.3 & 8.3 & 3.5 \\
223 & 14.8 & 8.4 & 3.6 \\
\hline
\end{tabular}

well as $\Delta \alpha$ are in good agreement with the corresponding values of parent systems like poly(dimethyl siloxane) [37].

According to these results, the intersection temperature can be assigned to the glass transition temperature of the polymer. The simulated value of $210 \mathrm{~K}$ is also in excellent agreement with the experimental glass transition temperature [38]. A detailed discussion will be made further.

\subsection{Chain dimensions}

Table 1 shows the end-to-end distance, radius of gyration and characteristic ratio of the poly(3-aminopropyl methyl siloxane) at the seven temperatures. The estimated characteristic ratio at room temperature, extrapolating the characteristic ratios obtained at temperatures over the glass transition temperature, was around 6 . This result agrees with experimental values of 57 expected for unperturbed chains of poly(dimethyl siloxane), of 67

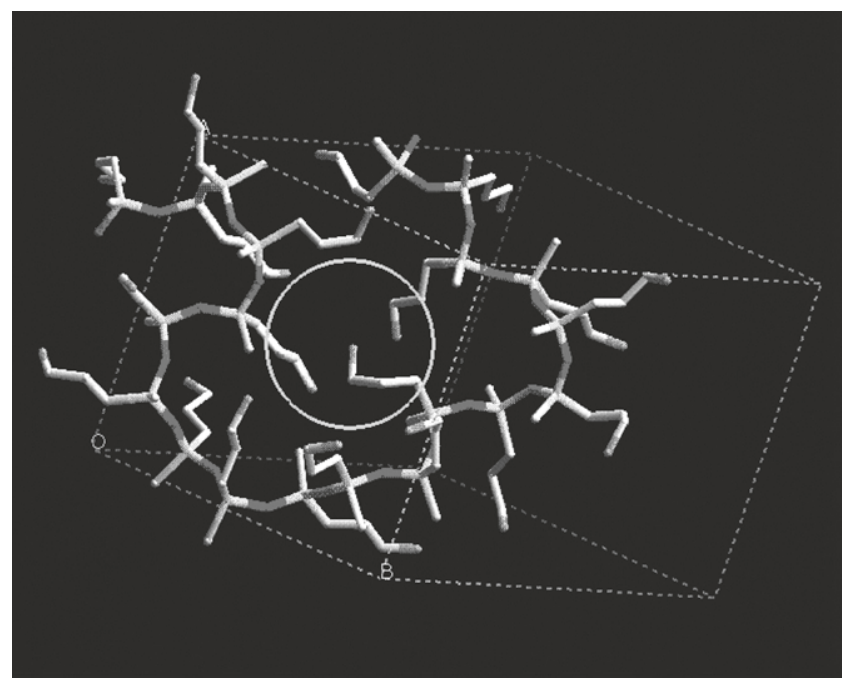

Fig. 2. Poly(3 aminopropyl methyl siloxane) shape in the polymer bulk system. 


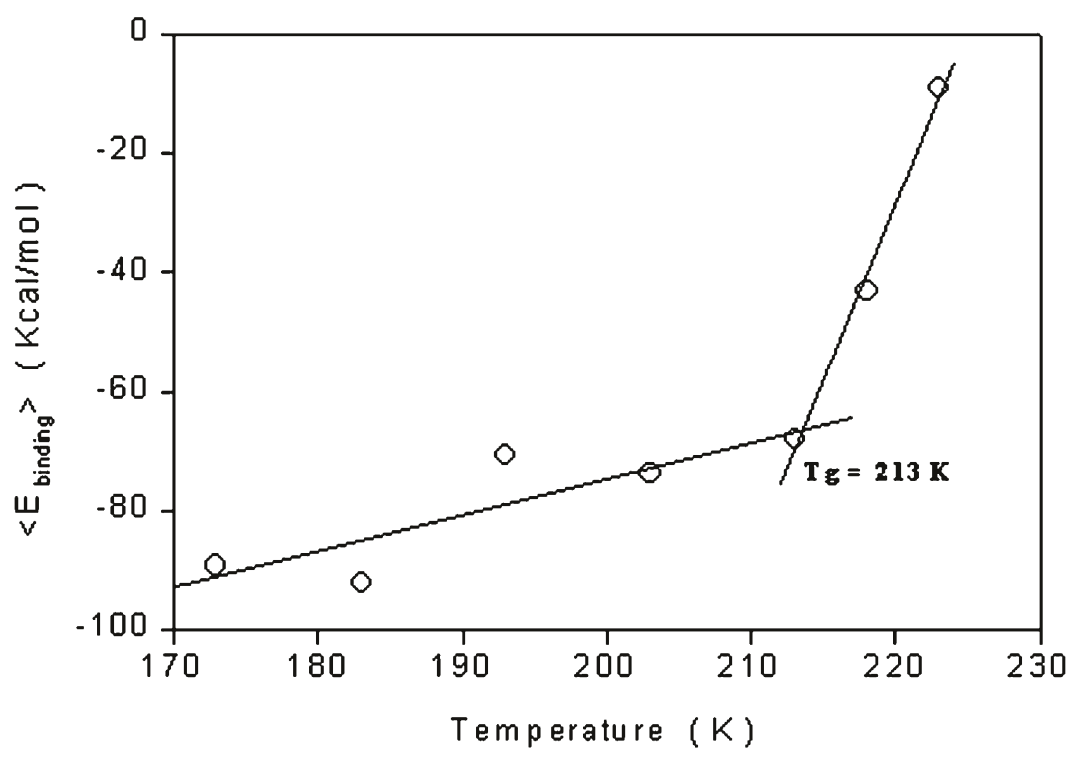

Fig. 3. Binding energy of poly(3 aminopropyl methyl siloxane) as a function of temperature.

expected for and poly(methylphenyl siloxane) $\left(\begin{array}{ll}10 & 14\end{array}\right)$ with a high number of bonds [39].

The polymeric shape of this system is presented in Fig. 2. The polymer system adopts conformations with the chains close to it. Three amino groups of the propylene chain are located near to the chain mass center and the rest of the amino groups are situated outside the main chain. The three amino groups located near the interior of the chain stabilize close conformations, and therefore the globular formation probability increases with respect to polymers like poly( $\gamma$-trifluoropropylmethyl siloxane) and poly(dipropyl siloxane).

\subsection{Energy interactions}

$E_{\text {binding }}$ between chains for the seven temperatures are represented in Fig. 3. $E_{\text {binding }}$ presents two different zones around $210 \mathrm{~K}$. Stabilization increases slowly for temperatures lower than $210 \mathrm{~K}$ and strongly for higher temperatures; this behavior is similar to that presented in Fig. 1. It is possible to confirm that for this low molecular weight polymer, the glass transition temperature depends strongly on the interactions between chains. The intersection of slopes defines a temperature of $213 \mathrm{~K}$ near the experimental glass transition temperature. The higher fluctuation of $E_{\text {binding }}$ for temperatures lower than $210 \mathrm{~K}$ is related to the experimental behavior observed in this polymer. Experimentally, it presents the glass transition temperature at $208 \pm 2 \mathrm{~K}$ and a small transition at a lower temperature. We cannot define the latest by molecular dynamics simulation, as the sensitivity of this method is too small for determining this point perfectly. Nevertheless, one of the causes for the high fluctuations at temperatures below $210 \mathrm{~K}$ could be the presence of the other transition experimentally observed.

The stabilization forces between chains are a very important contribution to the glass transition temperature. Van der Waals and electrostatic energy contributions to the binding energies are presented in Table 2. The most important contribution for the glass transition temperature was the electrostatic stabilization. This binding contribution energy increases with the temperature. For temperatures between 218 and $223 \mathrm{~K}$ the electrostatic contribution to the $E_{\text {binding }}$ presents positive values. The shape of the polymer chain is close to itself with approximately three amino groups near the polymer center of mass and the rest situated outside. The amino groups located near the exterior produce interactions that stabilize the material, for example hydrogen bonds. These interactions stabilize the polymer at low temperatures. For temperatures above the glass transition, the interactions like the hydrogen bonds between the amino groups have crashed as the positive values of the electrostatic contributions demonstrate. The values of the

Table 2

$E_{\text {binding }}$ and energy contributions of the polymer matrix for all temperatures

\begin{tabular}{llll}
\hline Temperature $(\mathrm{K})$ & $E_{\text {binding }}(\mathrm{kcal} / \mathrm{mol})$ & Van der Waals part $(\mathrm{kcal} / \mathrm{mol})$ & Electrostatic part $(\mathrm{kcal} / \mathrm{mol})$ \\
\hline 173 & -89.10 & -60.64 & -28.46 \\
183 & -92.12 & -69.34 & -22.77 \\
193 & -81.74 & -63.41 & -18.33 \\
203 & -71.98 & -59.72 & -12.19 \\
213 & -68.02 & -66.69 & -1.33 \\
218 & -42.96 & -60.71 & 17.75 \\
223 & -8.99 & -61.47 & 52.48 \\
\hline
\end{tabular}




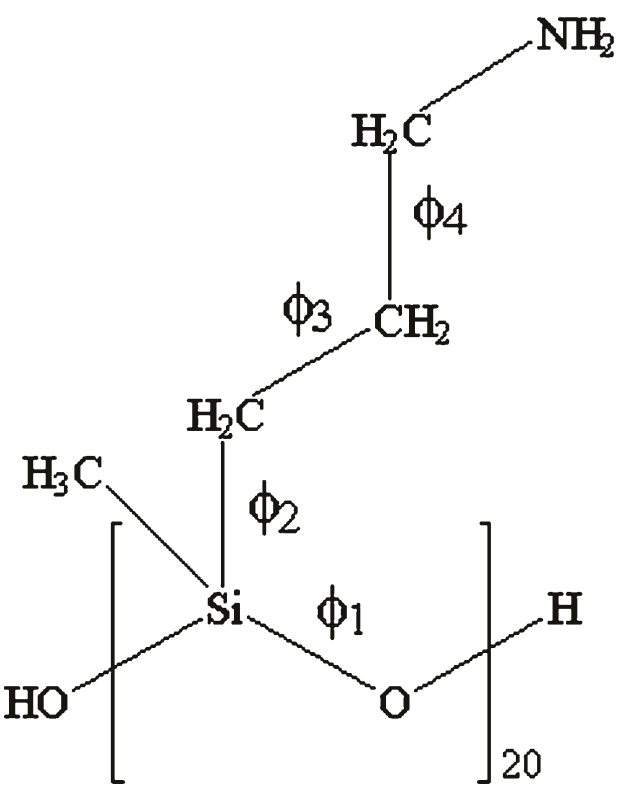

Fig. 4. Dihedral angles of poly(3 aminopropyl methyl siloxane).
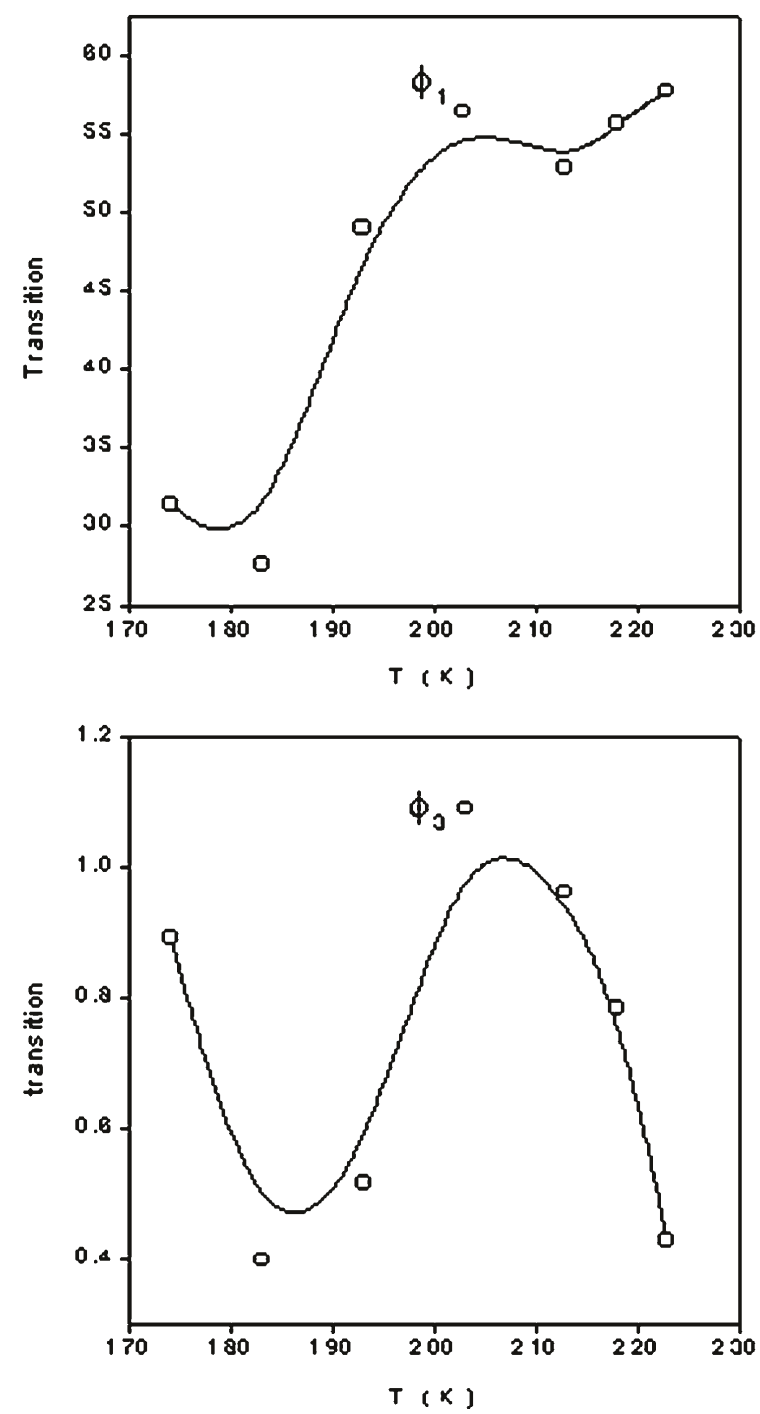

hydrogen bond contributions cannot be calculated with the PCFF force field because this contribution is included in the Van der Waals and electrostatic contributions. The Van der Waals contributions in the volume changes are less important than the electrostatic ones.

\subsection{Chain mobility}

Four torsion angles were evaluated, $\phi_{1}, \phi_{2}, \phi_{3}$ and $\phi_{4}$ (Fig. 4). These angles present the three classical states, trans $180^{\circ}$ and $g^{ \pm} \pm 60^{\circ}$. The chain mobility was evaluated by the transition between these three states. Fig. 5 shows the total transition torsion angle variation with the temperature. The mobility of angles $\phi_{3}$ and $\phi_{4}$ is less important than that of angles $\phi_{1}$ and $\phi_{2}$, because the number of transitions between states is too small to define the system mobility. Angles $\phi_{1}$ and $\phi_{2}$ present two different behaviors:

1. Angle $\phi_{1}$ increases strongly with the temperature starting from $185 \mathrm{~K}$. For temperatures higher than $185 \mathrm{~K}$ the
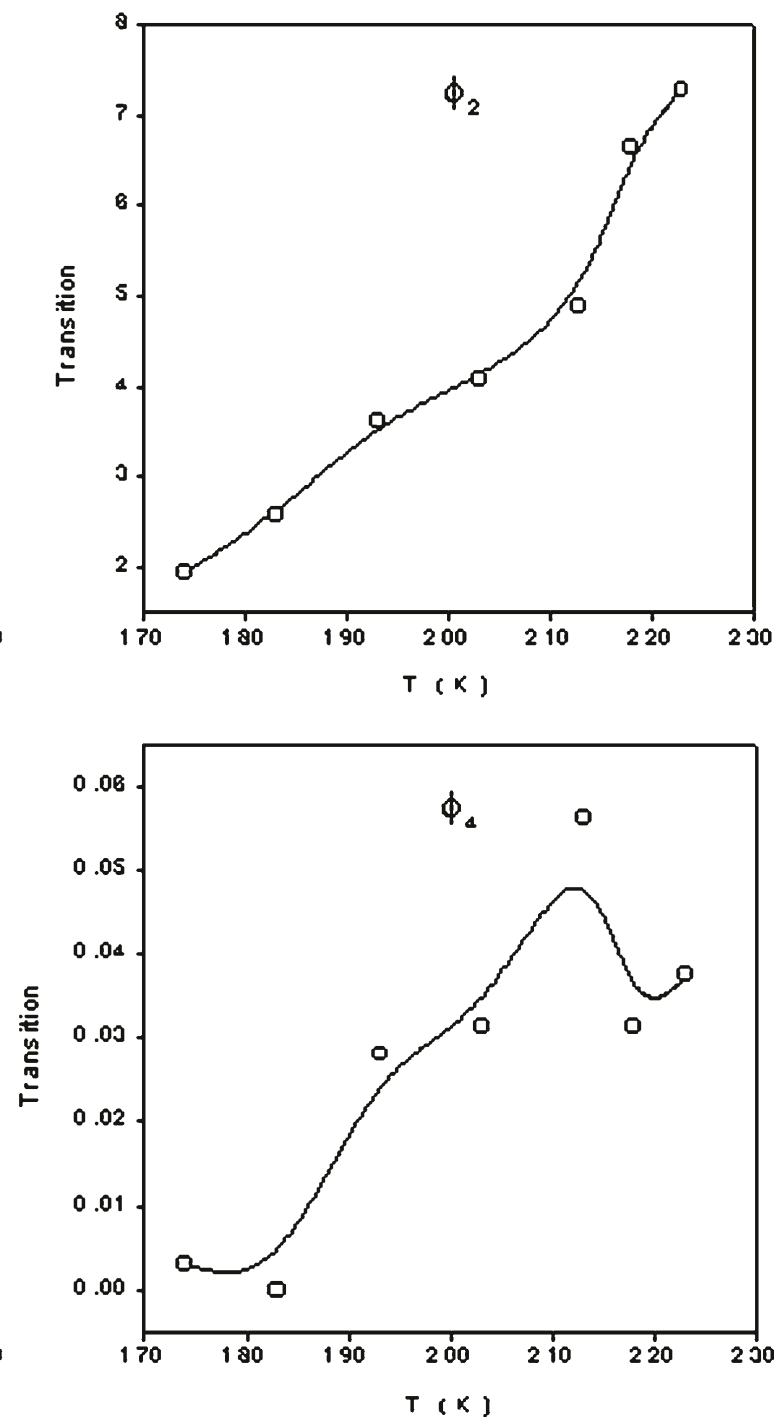

Fig. 5. Rotational transition number per bond between trans, $g^{+}$and $g$ states on poly( 3 aminopropyl methyl siloxane) $\phi_{1}, \phi_{2}, \phi_{3}$ and $\phi_{4}$ torsion angles. 


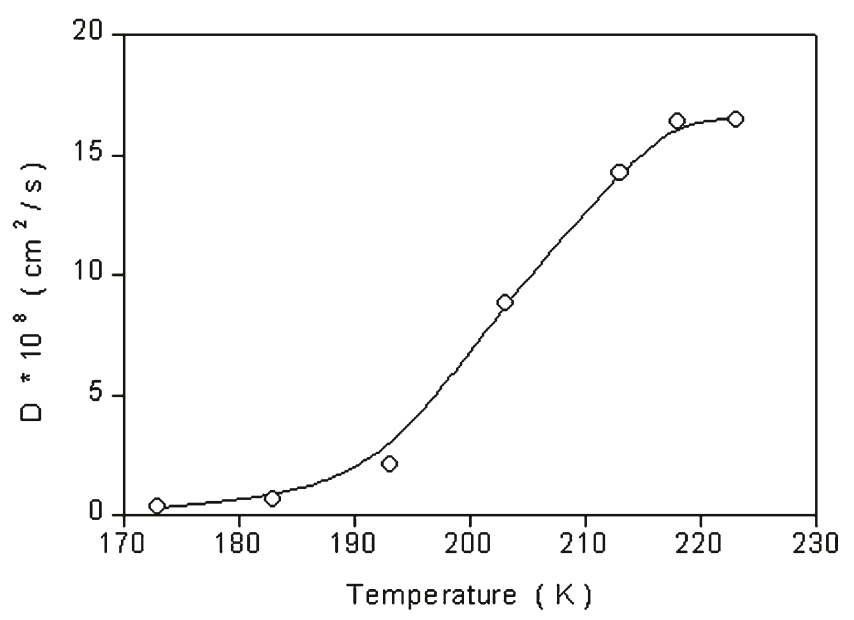

Fig. 6. Poly(3 aminopropyl methyl siloxane) Self diffusion constants as a function of temperature.

mobility of the siloxane chain increases strongly and presents the highest mobility for temperatures around $210 \mathrm{~K}$. This characteristic should be related with the glass transition temperature of the polymer chain.

2. Angle $\phi_{2}$ increases strongly with the temperature starting from $213 \mathrm{~K}$. The lateral chain mobility increases sharply to a temperature higher than the alpha transition.

Related with the experimental results, we can confirm that the glass transition temperature experimentally observed is due to the cooperative rotational movements of two different angles, the $\mathrm{O} \mathrm{Si}$ and $\mathrm{Si} \mathrm{C}$ torsion angles. We can conclude that the glass transition temperature observed experimentally is due to the cooperative movement of polymer chains originated by the torsion mobility of $\mathrm{O} \mathrm{Si}$ bonds of the principal chain and the $\mathrm{Si} \mathrm{C}$ bonds of the lateral chains.

\subsection{Self-diffusion constant of all atoms}

The variation of all polymer atoms of MSD with temperature was calculated. The self-diffusion constant (Eq. (3)) was obtained from MSD vs. time slope (Eq. (3)). Fig. 6 shows the self-diffusion constant of all the atoms of the poly(3-aminopropyl methyl siloxane) chain. This constant presents an increase with the temperature that is similar to observed in the transition number between the $\mathrm{Si} \mathrm{O}$ torsion bond stable states (Fig. 5). This result shows that cooperative movement of the polymer chain starts at temperatures around the glass transition temperature.

\section{Conclusions}

The intersection of slopes of the average specific volume of poly(3-aminopropyl methyl siloxane) as a function of temperature define the glass transition temperature at $210 \mathrm{~K}$, a value similar to that of the experimental result. Globular shaped polymer chains were observed with the molecular dynamics simulations. The chain is closed itself, three amino groups of the propylene chain are situated near the center and the rest of the amino groups of amino propyl chains are located outside the main chain. This low molecular weight polymer strongly depends on interactions between chains. The intersection of slopes defines a temperature of $213 \mathrm{~K}$ near the glass transition temperature. The most important contribution for the glass transition changes is the electrostatic binding energy contribution. The van der Waals contributions are less important.

The mobility chain was evaluated by the transition between the states trans, $g^{+}$and $g$ of torsion angles. The $\phi_{1}$ and $\phi_{2}$ angles present two different behaviors. The $\phi_{1}$ angle increases strongly with the temperature starting from $185 \mathrm{~K}$. For temperatures higher than $185 \mathrm{~K}$ the mobility of the siloxane chain is higher than for lower temperatures. This characteristic should be related with the glass transition temperature of the polymer chain. Angle $\phi_{2}$ increases strongly with the temperature starting from $213 \mathrm{~K}$. The lateral chain mobility increases strongly with temperatures higher than that of the main chain. In relation to the experimental results we can confirm that the glass transition temperature observed experimentally, $208 \pm 2 \mathrm{~K}$ [38], is due to the rotational mobility of two different angles: $\mathrm{O} \mathrm{Si}$ and $\mathrm{Si} \mathrm{C}$. The self-diffusion constant variation of all polymer atoms with the temperature is another piece of evidence that demonstrates that the glass transition temperature is due to the cooperative rotational mobility of these torsion angles.

\section{Acknowledgments}

This work was supported by the CAM through Grant 07N/0002/1998. 


\section{References}

[1] Mittal KL. Silanes and other coupling agents, Utrech 1992.

[2] Wang D, Jones FR, Denison P. J Mater Sci 1992;27:36.

[3] Wang D, Jones FR, Denison P. J Mater Sci 1993;28:2481.

[4] González Benito J, Baselga J, Aznar AJ. J Mater Process Technol 1999;92 93:129.

[5] Ishida H. Polym Compos 1984;5:101.

[6] Thomason JL, Dwight DW. Composites Part: A 1999;30:1401.

[7] Kim JK, Mai YW. Interfaces in composites. In: Chou TW, editor. Structure and properties of composites, vol. 13. Weinheim: $\mathrm{VCH}$; 1993.

[8] González Benito J, Aznar AJ, Lima J, Bahía F, Maçanita AL, Baselga J. J Fluoresc 2000;10:141

[9] Rigby D, Roe RJ. J Chem Phys 1987;87:7285.

[10] Sylvester MF, Yip S, Argon AS. Nomenclature. In: Roe RJ, editor. Computer simulation of polymer. Englewood Cliffs, NJ: Pretice Hall; 1991.

[11] Takeuchi H, Roe RJ. J Chem Phys 1991;94:7458.

[12] Roe RJ, Rigby D, Furuya H, Takeuchi H. Comput Polym Sci 1992;2: 32.

[13] Roe RJ. J Chem Phys 1994;100:1610.

[14] Roe RJ. Adv Polym Sci 1994;116:111.

[15] Pant PVK, Han J, Smith GD, Boyd RH. J Chem Phys 1993;99:597.

[16] Boyd RH, Gee RH, Han J, Him YJ. J Chem Phys 1994;101:788.

[17] Han J, Gee RH, Boyd RH. Macromolecules 1994;27:7781.

[18] Fan CF, Çagin T, Shi W, Smith KA. Macromol Theory Simul 1997;6: 103.

[19] Boyer RF. Polym Engng Sci 1968;8:161.

[20] Theodorou DN, Suter UW. Macromolecules 1985;18:1467.

[21] Dinur U, Hagler AT. New approaches to empirical force fields. Reviews in Computational Chemistry; 1991. vol 2 Lipkwowitz KB, Boyd DB (editors). VCH:New York. Chapter 4.

[22] Maple JR, Dinur U, Hagler AT. Proc Natl Acad Sci USA 1998;85: 5350.

[23] Hwang MJ, Stockfisch TP, Haggler AT. J Am Chem Soc 1994;116: 2515.

[24] Sun H, Mumby SJ, Maple JR, Hagler AT. J Am Chem Soc 1994;116: 2978.

[25] Sun H, Mumby SJ, Maple JR, Hagler AT. J Phys Chem

1995;99:5873.[26] Sun H. Macromolecules 1995;28:701.

[27] Sun H. Macromolecules 1994;26:5924

[28] Sun H. J Comput Chem 1994;15:752.

[29] Andersen HC. J Chem Phys 1980;72:2384.

[30] Parrinello M, Rahman A. J Appl Phys 1981;52:7182.

[31] Nose' S. J Chem Phys 1984;81:511.

[32] Nose' S. Mol Phys 1984;52:511.

[33] Hoover WG. Phys. Rev. A 1985;31:1695.

[34] ,Cagin T, Goddard WA, Ary ML. Comput Polym Sci 1991;1:241.

[35] Cerius2 4.2, Molecular Simulations Inc., San Diego, USA.

[36] Ferry JD. Viscoelastic properties of polymers. New York: Wiley; 1980. p. 281.

[37] Barlow AS, Harrison G, Lamb J. Proc R Soc 1964;228:1282, listed in Ref. [38].

[38] Cabanelas JC, Serrano B, Gonzalez Benito J, Bravo J, Baselga J. Macromol Rapid Commun 2001;22:694.

[39] Polymer Handbook. Wiley Interscience, 1999. 